\title{
An alternative method to calculate cross-flow instabilities
}

\author{
Soledlad Le Clainche ${ }^{* \dagger}$, Meng-Meng Wu ${ }^{\ddagger}$, Zhong-Hua Han ${ }^{\S}$, Esteban Ferrer
}

\begin{abstract}
This work presents a new method to compute instabilities associated to cross-flow transition scenarios. The method analyses base flows issued from CFD simulations (RANS model) using higher order dynamic mode decomposition (HODMD). The flow field is then approximated as an expansion of modes and it is possible to identify the primary and secondary flow instabilities, in cross-flows. The good performance of this novel method is first validated by locating the transition point related to the first instability in a low-speed 2D NLF0416 airfoil. Then, 3D cross-flow instabilities over a wing with $40^{\circ}$ back-sweep angle (shaped from a NACA642A015 airfoil) are presented.
\end{abstract}

\section{Introduction}

Commercial aircraft include relatively large sweep angles on the wings, such that transonic effects are minimised at cruise flow conditions. When the sweep angle is small, Tollmien-Shlighting (TS) waves govern the transition mechanism (as in 2D airfoils) in boundary layers. This viscous instability was first theoretically shown by Tollmien (1929) and Schlichting (1933). TS waves develop in boundary layers and grow exponentially in space (but are temporally stable) until non-linear interactions dominate leading to the turbulent regime.

For large sweep angles, the mean flow outside the boundary layer curves leading to a secondary flow and an inflection point (Saric et al. 2003). As a consequence, the primary instability is a low-frequency travelling crossflow, which saturates to lead to steady spanwise modulations (i.e a periodic spanwise flow). In low-disturbance environments, a high-frequency secondary instability develops upon the cross-flow shear-layer vortices resulting from the first instability, dominate the transition mechanism. Typically, these secondary instabilities show a high frequency signature and an rapid spatial growth (when compared to $\mathrm{TS}$ growth). These secondary instabilities dominate cross-flow transition scenarios over swept wings.

Researchers have studied cross-flow transition by means of various techniques. Local linear stability analysis and the eN method have been successful on predicting TS type transition, but the underlying linearity assumption has limited its application to predict the onset of turbulent flows in complex configurations (e.g. cross-flow transition scenarios). An alternative is to use more sophisticated techniques, such as 2D eigenfunctions [8], Floquet analysis or linear and nonlinear Parabolized Stability Equations (PSE), see e.g.[9]. However, these techniques may find difficulties in predicting mode interactions.

In this article, we present a method that can be applied to analyse secondary flow instabilities when non-linear effects are non-negligible, such that not only the primary flow instabilities are identified, but also secondary interactions. The method relies on performing a base flow simulation where the transition point is fixed. Aft this point, a turbulent model is utilised to avoid the necessity of resolving turbulent structures, whilst before the transition point a fine enough grid is used to compute a direct simulation (no modelling) of the instabilities governing transition. The simulation of the laminar region is not computationally expensive but includes the information of small disturbances that can grow spatially to govern the transition mechanism. In particular TS and cross-flow instabilities may be determined. These structures are extracted from the CFD simulations using a higher order dynamic mode decomposition (HODMD) technique [3], applied to spanwise and streamwise spatial directions, to calculate the wavenumber associated with spatial leading instabilities. The main advantage of using this technique lies in the capability of HODMD to represent the flow dynamics in fully three-dimensional data, without the need of any type of simplification much as parallel assumptions.

\section{Higher order dynamic mode decomposition}

HODMD is a method that decomposes spatio-temporal data $\mathbf{v}_{k}$ as an expansion of modes in the following way:

\footnotetext{
*Correspondence to: soledad.leclainche oupm.es.

${ }^{\dagger}$ Lecturer in Applied Mathematics. School of Aerospace Engineering, Universidad Politécuica de Madrid, E-28040, Madrid, Spain.

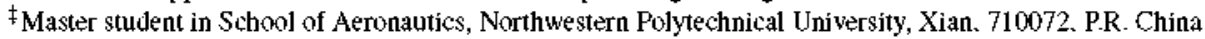

$\S$ Professor in School of Aeronautics, Northwestern Polytechnical University, Xian, 710072, P.R. China

LLecturer in Applied Mathenatics, School of Aerospace Engineering, Universidad Politécuica de Madrid, E-28040, Madrid, Spain.
} 


$$
\mathbf{v}_{k} \simeq \mathbf{v}_{k}^{D M D} \equiv \sum_{m=1}^{M} a_{m} \mathbf{u}_{m} e^{\left(\delta_{m}+i \omega_{m}\right),},
$$

where $\mathbf{u}_{m}$ represents the Dynamic Mode Decomposition (DMD) modes, and $a_{m}, \omega_{m}$ and $\delta_{m}$ are their corresponding amplitudes, frequencies and temporal growth rates. The method can also be applied to a set of data along a spatial component to calculate the wavenumbers $\beta_{n}$ of the most relevant spatial (in the remaining directions) or temporal modes $\mathbf{u}_{n}$, and associated spatial growth (or decay) rates $v_{n}$. Equation (1) may be re-written for spatial decompositions as

$$
\mathbf{v}_{k}=\sum_{n=1}^{N} a_{n} \mathbf{u}_{n} e^{\left(v_{n}+i \beta_{n}\right) x} .
$$

The original spatio-temporal HODMD algorithm is explained in detail in [3]. It considers two main steps. As first step, the spatial redundancies (such as noise [3] or transient modes [4]) are removed via a Singular Value Decomposition (SVD) filtering. Based on a certain tolerance (set by the user) $\varepsilon_{S V D}$, only the most relevant SVD modes are retained. In a second step, the method combines DMD $[1,10]$ with the Takens' embedding theorem [11] , using $d$ delayed snapshots. This algorithm is known as DMD-d. When $\mathrm{d}=1$, the algorithm recovers the original DMD technique [10], but for values of $d>1$, the robustness of the method is improved to analyse complex flows, noisy data or transitional flows $[2-4,7]$. The final number of retained DMD modes is set according to a certain tolerance $\varepsilon$, that ranks the mode amplitudes.

When the data analyzed is significantly noisy, the algorithm is applied iteratively to remove spurious modes. This last modification is retained for our study. Additional details may be found in [5].

It is possible to obtain spatio-temporal expansions, if HODMD algorithm is applied sequentially as in [6]. Similarly, it is possible to obtain expansions related to two spatial directions

$$
\mathbf{v}_{k}=\sum_{m=1, n=1}^{M, N} a_{m n} \mathbf{u}_{m n} e^{\left(v_{n}+i \beta_{n}\right) x} e^{\left(\delta_{m}+i \kappa_{m}\right)} .
$$

In this article we study cross-flow instabilities by applying the sequential method to the streamwise and spanwise components. Sequential spatial HODMD consists in three main steps.

1) We apply HODMD along the spanwise direction to obtain expansions of the steady spanwise modes characterising cross-flow instabilities

$$
\mathbf{v}_{k}=\sum_{m=1}^{M} a_{m} \widehat{\mathbf{u}}_{m} e^{\left(\delta_{m}+i k_{m}\right) z} .
$$

2) Subsequently, we apply HODMD along the streamwise direction to the modes obtained in the previous expansion as

$$
\widehat{\mathbf{u}}_{m}=\sum_{n=1}^{N} \tilde{a}_{m n} \mathbf{u}_{m n} e^{\left(v_{m n}+i \beta_{m n}\right) x} .
$$

3) Calling $a_{m n}=a_{m} \cdot \ddot{a}_{m n}$, and introducing expansion (4) into (5), one easily obtains the original expansion (3).

The sequential method is the method applied in this article. This expression is suitable to study secondary cross-flow instabilities. Global instabilities are obtained studying the variations along the streamwise component of the amplitudes calculated in equation (5).

The $\mathrm{N}$ factor, usually calculated in linear theory, is approximated with the evolution of the mode amplitudes along the spanwise direction (we perform several analyses in this direction as described in Section IV) and approximated as $N_{i} \simeq \log \left(a_{i} / a_{0}\right)$, being $a_{0}$ the highest amplitudes of the DMD expression (5) presented before.

\section{Numerical simulations and validations}

Numerical simulations have been performed using an in-house CFD solver, called "PMNS2D". Three-dimensional compressible Reynolds-Averaged Navier-Stokes (RANS) equations have been solved using Jameson-Schmidt-Turkel scheme with artificial dissipation for the spatial discretisation and an LU-SGS scheme for time integration, with a multigrid technique to accelerate the iterative convergence to steady state. The formal spatial accuracy is of second order. 
First, numerical simulations have been carried out to study a 2D low-speed laminar NLF0416 airfoil. The base flow is obtained by solving the Navier-Stokes equations under a prescribed transition point $(x / c=0.6)$ at a freestream Mach number of 0.1 , and Reynolds number of $4 \times 10^{6}$ (based on the airfoil chord). The C-mesh for the $2 \mathrm{D}$ case is similar taking a section of the 3D meshes used later for the cross-flow computations. The distance between the first grid and the airfoil surface is $1 \times 10^{6} \mathrm{c}$, where $\mathrm{c}$ denotes the chord, such that $y+<1$. The typical grids have 640 points in the streamlined direction ( 512 points on the airfoils, 256 on suction and pressure sides) and 240 points in the direction normal to the airfoil surface. Second, a 3D NACA642A015 wing is studied at a Reynolds number $R e=7 \cdot 10^{6}$, based on the wing chord. The wing is generated by extruding a NACA642A015 airfoil with a back-sweep angle of $40^{\circ}$. The $3 \mathrm{D}$ mesh has $352 \times 104 \times 24$ elements (a total of 878.592 ). To discretise the spanwise direction we have used 24 planes, which are enough to discretise the long wavelength spanwise oscillation, characteristics of cross-flows over swept wings. A mesh is shown in Figure 1 for sweep angle $40^{\circ}$, together with the streamlines on the wing and pressure contour on a plane parallel to the free stream velocity.

a)

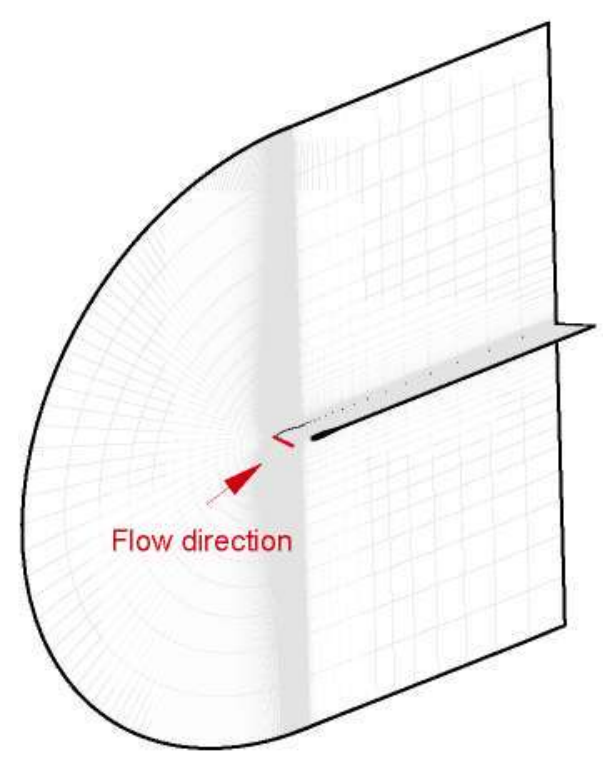

b)

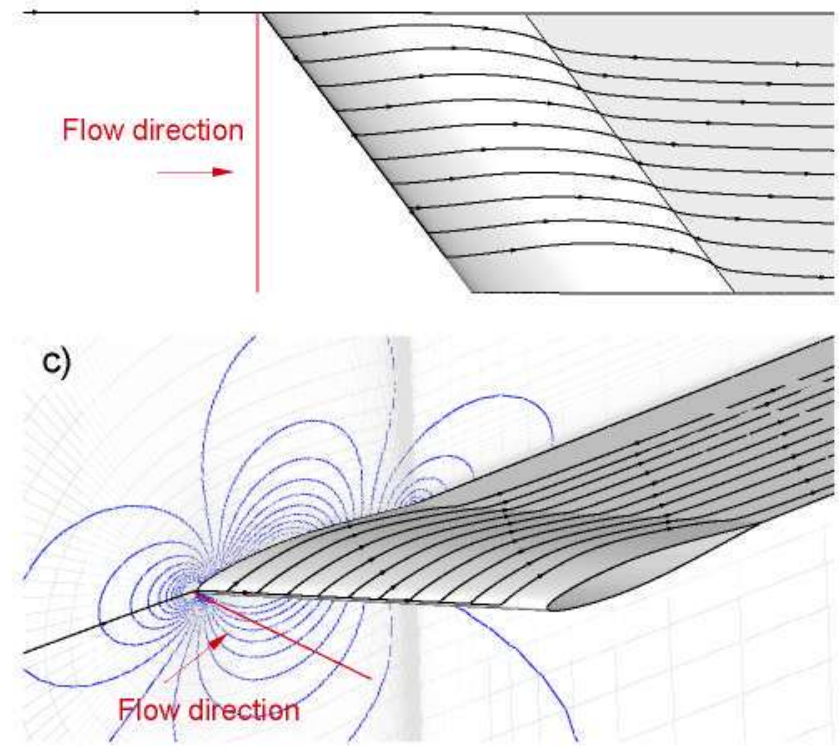

Fig. 1 a) Computational domain for the steady base flow with sweep angle $\Lambda=40^{\circ}$, b) top view wing streamlines on the wing and c) near airflow mesh and contour of static pressure (blue lines).

\section{Transition point in a two-dimensional airfoil}

To validate the methodology proposed in this article, HODMD has been applied to capture the transition point of the 2D low-speed laminar NLF0416 airfoil. The method has been applied in the upper and lower surface of the body in 40 different sections $A_{i}$, located at $0.15<x / c<0.53$, shown in Figure 2.

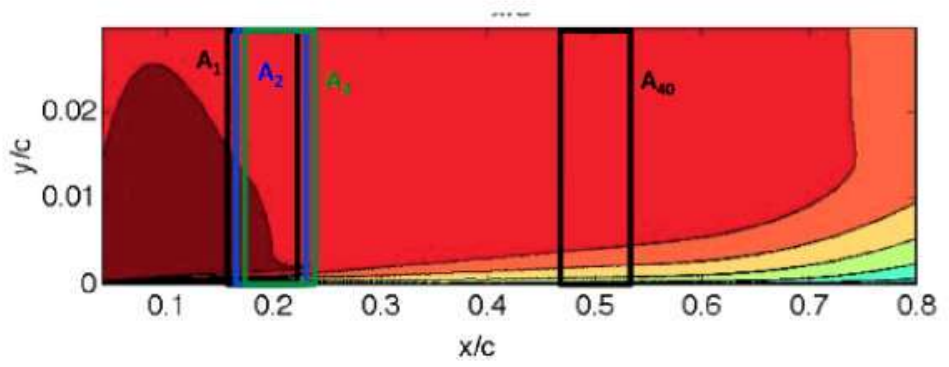

Fig. 2 Domain for the HODMD analysis in the upper surface of the 2D low-speed NLF0416 airfoil. 
The number of snapshots used in each section, equispaced in the streamwise direction, is 20. After some calibration, the parameters used for this analysis are $d=8$ and $\varepsilon_{S V D}=10^{-8}$ and $\varepsilon=10^{-4}$. Similar results are obtained using higher tolerances, for values of $\varepsilon_{S V D} \leq 10^{-5}$ and $\varepsilon \leq 10^{-3}$ and decreasing the number of snapshots to 10 (showing the robustness of this method). The method captures the wavenumber for the leading mode (as the highest amplitude mode) in the upper surface $\beta /(2 \pi) \simeq 44$, and $\beta /(2 \pi) \simeq 63$ in the lower surface. Figure 3 shows the spatial evolution of each one of these modes along the upper and lower surfaces. The figure shows the $\mathrm{N}$-factor related to each one of these flow instabilities. As seen, in the experimental results, presented in $[12,13]$, the flow transition occurs for $N \simeq 9$. In this case, the flow transitions at points $x / c=0.31$ and $x / c=0.52$ in the upper and lower surfaces, respectively. This result is in good agreement with experiments[12,13], that predict the transition points at $x / c \simeq 0.35-0.40$ and $x / c \simeq 0.5-0.55$ for the upper and lower surfaces, respectively.
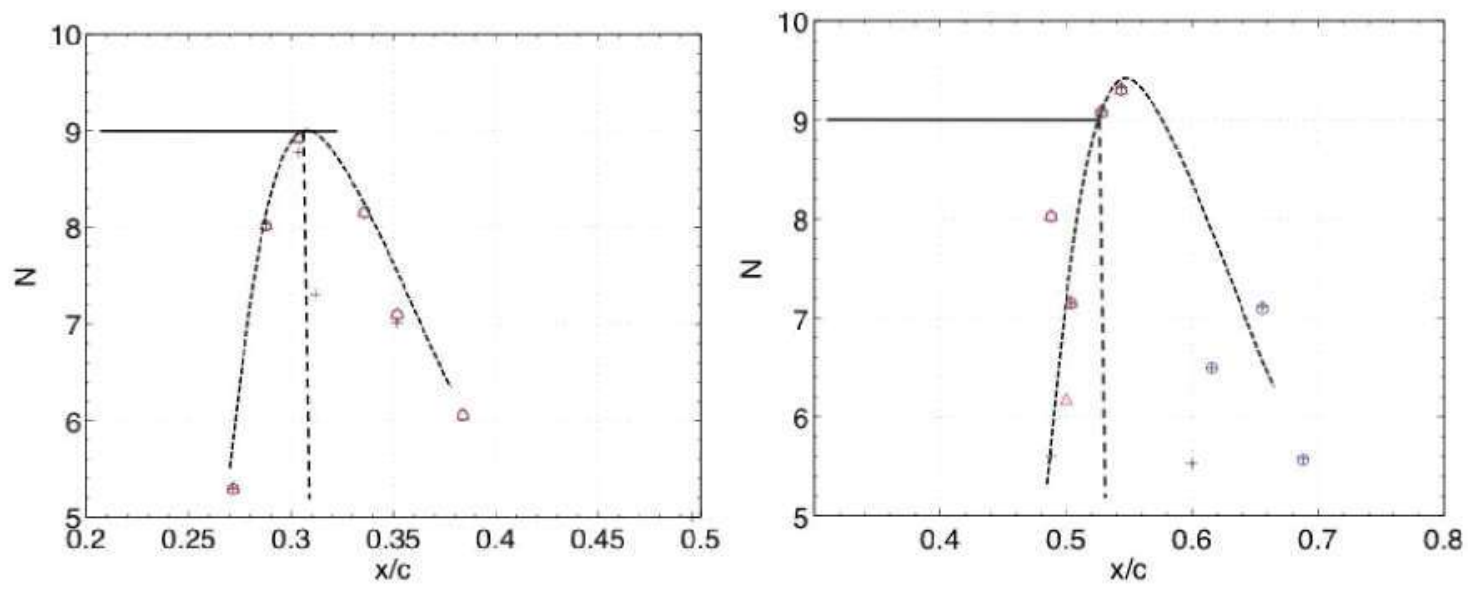

Fig. 3 Evolution of the amplitude of the leading mode as function of the streamwise component in the 2D NLF0416 airfoil. Left: upper surface. Right: lower surface. $\mathbf{N}$ factor calculated as $N=\log \left(a / a_{0}\right)$. The various symbols represent the results obtained using the following parameters with DMD-8: black crosses for $\varepsilon_{1}=10^{-6}$, $\varepsilon=10^{-4}$ using 20 snapshots; blue circles for $\varepsilon_{1}=10^{-4}, \varepsilon=10^{-4}$ using 20 snapshots; red triangles for $\varepsilon_{1}=10^{-4}$, $\varepsilon=10^{-4}$ and using 10 snapshots.

\section{Cross-flow instabilities}

The prediction of a cross-flow instability in a 3D NACA642A015 airfoil is presented in this section. The methodology used for this analysis is similar to the one presented in the two-dimensional case. However, in this case it is necessary to calculate the global modes related to the spanwise direction too. Thus, the sequential spatial HODMD is applied, first along the spanwise direction, and next along the streamwise direction.

An example of the base flow obtained for a sweep angle $\Lambda=40$ is shown in Figure 4 , where a periodic spanwise flow can be seen in Figure 4 a) Using DMD, we will seek secondary perturbation upon this spanwise periodic flow.

As first step of the sequential spatial HODMD method, we perform the analysis along the spanwise direction of the flow. As expected, the flow is found spatially periodic, with dominant wavenumber $k /(2 \pi)=1$. The parameters used in this analysis are $1 \leq d \leq 5$, while the tolerances are set to machine error with the aim at obtaining the larger accuracy in the calculations, $\varepsilon_{S V D}=\varepsilon=10^{-8}$. The number of spatial points used in the analysis are 24 , equidistant in space with $\Delta z=4.17 \cdot 10^{-2}$.

In order to predict the transition point linked to this spanwise mode, we apply HODMD along the streamwise direction. As explained before, HODMD is applied into the dominant spanwise mode with $k /(2 \pi)=1$. The methodology followed to depict the transition point is also based in the variations of the amplitudes, and is the same as the one explained in Section IV (the same sub-domains $A_{i}$ are used in this calculations with the tolerances $\varepsilon_{S V D}=\varepsilon=10^{-8}$ ).

Figure 5 shows the results obtained. The transition point predicted by the experiment is $x / c \simeq 0.33$ for a value of $\mathrm{N}=7.5$. In the figure it is possible to see that the method predicts, for the DMD with highest amplitude, the transition point at $x / c \simeq 0.3$ for $\mathrm{N}=7.5$, which is in good agreement with the experimental result. 
a)

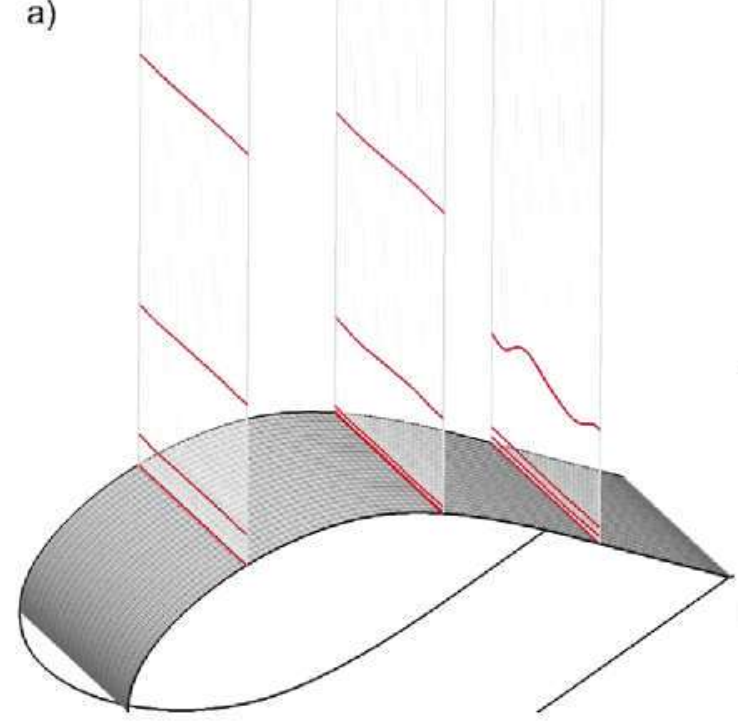

b)

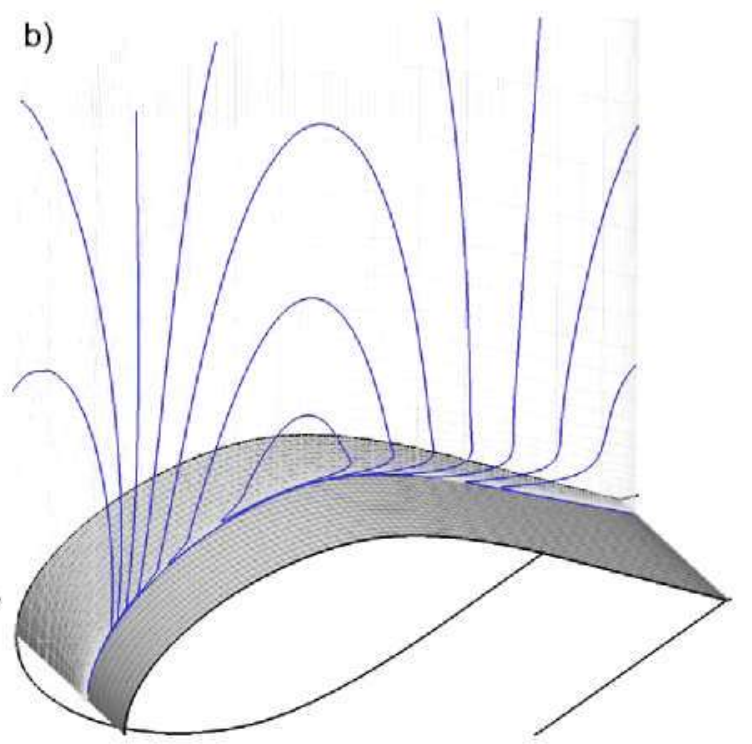

Fig. 4 Base flow for Sweep angle $\Lambda=40^{\circ}$ : W-velocity contours for a) planes parallel to the leading edge and b) following the free stream velocity direction.

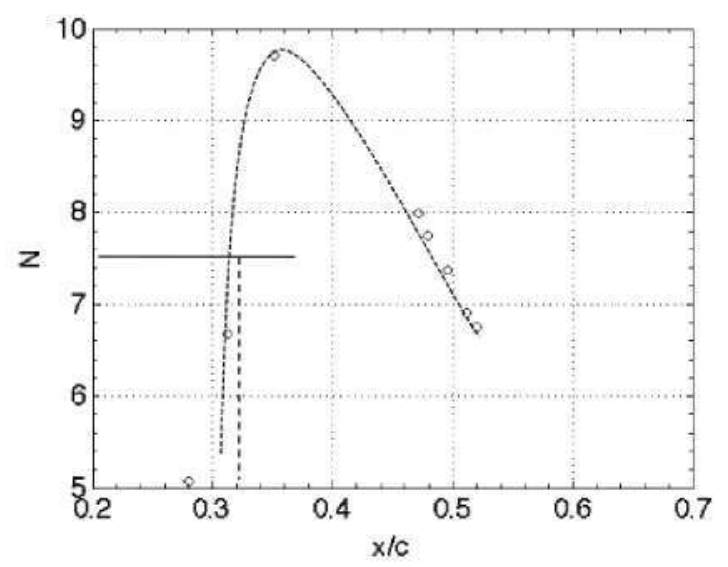

Fig. 5 Counterpart of Figure 3 for the 3D cross-flow leading instability for a wing with sweep angle $40^{\circ}$ and $\mathbf{R e}=7 \cdot 10^{6}$. DMD-8 with tolerances $\varepsilon_{1}=10^{-4}, \varepsilon=10^{-4}$.

\section{Conclusions}

We have presented a new method to study cross-flow instabilities. The method is based on a sequential spatial HODMD. The main advantage is that this data-driven method can be applied to analyze non-linear flows, opening new horizons for analyzing cross-flow instabilities coming from experimental measurements (i.e.: time-resolved tomo-particle image velocimetry).

In the article the method has been validated to predict the transition point in a two dimensional profile. Then, the method has been successfully applied to predict the transition point from a cross-flow instability. The parameters used in both analyses are the same, showing that the method is robust. The results are compared with experimental data, showing favourable comparisons. 


\section{References}

[1] Ferrer, E., DeVicente. J. \& Valero. E. 2014 Low cost 3D global instability analysis and flow sensitivity based on dynamic mode decomposition and high-order numerical tools Int. J. of Num. Meth. in Fluids 76 (3), 169-184.

[2] Le Clainche, S., Sastre, F., Vega, J.M., Velazquez, A. 2017 Higher order dynamic mode decomposition applied to study flow structures in noisy PIV experimental data AIAA 2017-3304, 47th AIAA Fluid Dynamics Conference DOI: 10.2514/6.2017-3304

[3] Le Clainche. S. \& Vega. J.M. 2017 Higher Order Dynamic Mode Decomposition. SIAM J. on Appl. Dyn. Systems 16(2). 882-925

[4] Le Clainche. S. \& VeGA, J.M. 2017 Higher order dynamic mode decomposition to identify and extrapolate flow patterns. Physics of Fluids $29(8) 084102$

[5] Le Clainche, S., Vega, J.M., \& Soria, J. 2017 Higher order dynamic mode decomposition of noisy experimental data: the flow structure of a zero-net-mass-flux jet Exp. Therm. and Fltid Sci. 88, 336-353

[6] Le Clainche, S., PÉrez, J.M. \& Vega, J.M. 2018 Spatio-temporal flow structures in the three-dimensional wake of a circular cylinder. Fluid Dynamics Research DOI: 10.1088/1873-7005/aab2f 1

[7] Le Clainche. S., Ferrer, E. 2018 A Reduced Order Model to Predict Transient Flows around Straight Bladed Vertical Axis Wind Turbines. Energies 11(3)-566

[8] MaLIK, M. R., L. F., \& ChanG, C.-L. 1994 Crossflow disturbances in three-dimensional boundary layers: nonlinear development. wave interaction and secondary instability $J$. Fluid Mech. 268, 1-36

[9] Paredes, P., Choudhari, M. M., AND Li, F. 2016 Nonlinear Transient Growth and Boundary Layer Transition AlAA Paper 2016-3956

[10] Schmid, P.J. 2010 Dynamic mode decomposition of numerical and experimental data. J. Fluid Mech. 656. 5-28.

[11] F. TAKens, Detecting strange attractors in turbulence. Lecture Notes in Mathematics. D.A. Rand and L.-S. Young. eds.. Springer-Verlag. 1981,) pp. 366-381.

[12] M.M Wu, Z.H Han, S.N Wang, W.P Song, E. Ferrer 2017 A DMD-Based Automatic Transition Prediction Method for Flows over Airfoils 47th AIAA Fluid Dynamics Conference. Denver, Colorado. AIAA 2017-4303

[13] Wu, M.M., Han.Z.H., Wang, S.N., Song, W.P., Fer rer, E. 2018 A Transition Prediction Method for Flows over Airfoils Based on Dynamic Mode Decomposition (Under review) 